\title{
Comparison of the Gaussian and Bessel Function Exchange Functionals with the Hartree-Fock Exchange for Molecules
}

\author{
Gregory J. Laming and Nicholas C. Handy* \\ Department of Chemistry, Cambridge University, Cambridge, CB2 IEW, England
}

William H. Miller

Department of Chemistry, University of California/Berkeley, Berkeley, California 94720

Received: July 18, 1994; In Final Form: October 24, $1994^{\otimes}$

\begin{abstract}
We present Kohn-Sham and hybrid density functional theory calculations on the hydrogen and nitrogen molecules using the Gaussian function exchange energy expression $K_{\mathrm{G}}$ derived by Ghosh and Parr. We compare the potential energy surfaces, equilibrium bond lengths, and exchange energies obtained by using $K_{\mathrm{G}}$ and related models with those predicted from Hartree-Fock theory and the local density approximation to the exchange. Such functionals do not appear to offer promising alternative starting points to the Dirac uniform electron gas exchange functional.
\end{abstract}

\section{Introduction}

With density functional theory (DFT) our aim is to calculate high-accuracy molecular properties. ${ }^{1}$ If we opt for the Kohn$\mathrm{Sham}^{2}$ DFT formalism, this amounts to solving the Kohn-Sham equations using the exact expression for the exchangecorrelation potential $v_{\mathrm{xc}}(\mathbf{r})$, the exchange-correlation potential $v_{x c}(\mathbf{r})$ being the functional derivative of the exchange-correlation functional $E_{\mathrm{xc}}[\varrho]$ :

$$
v_{\mathrm{xc}}(\mathbf{r})=\frac{\delta E_{\mathrm{xc}}[\varrho]}{\delta \varrho(\mathbf{r})}
$$

For a closed shell molecule the Kohn-Sham equations may be written as

$$
\begin{gathered}
{\left[-\frac{1}{2} \nabla^{2}+v(\mathbf{r})+\int \frac{\varrho\left(\mathbf{r}^{\prime}\right)}{\mid \mathbf{r}-\mathbf{r}^{\prime}} \mathrm{d} \mathbf{r}^{\prime}+v_{\mathrm{xc}}(\mathbf{r})\right] \psi_{i}(\mathbf{r})=\epsilon_{i} \psi_{i}(\mathbf{r})} \\
\varrho(\mathbf{r})=\sum_{i}^{N / 2} 2\left|\psi_{i}(\mathbf{r})\right|^{2}
\end{gathered}
$$

and the total energy $E[\varrho]$ is obtained by using the converged orbitals $\psi_{i}$ in the following equation:

$$
\begin{aligned}
& E[\varrho]=\sum_{i}^{N}\left|\psi_{i}\right|- \frac{1}{2} \nabla^{2}\left|\psi_{i}\right\rangle+\int v(\mathbf{r}) \varrho(\mathbf{r}) \mathrm{d} \mathbf{r}+ \\
& \frac{1}{2} \iint \varrho\left(\mathbf{r}_{1}\right) \frac{1}{r_{12}} \varrho\left(\mathbf{r}_{2}\right) \mathrm{d} \mathbf{r}_{1} \mathrm{~d} \mathbf{r}_{2}+E_{\mathrm{xc}}[\varrho]
\end{aligned}
$$

In practice we expand the Kohn-Sham orbitals $\psi_{i}$ in terms of a finite set of nonorthogonal basis functions $\eta_{\beta}$ such that

$$
\psi_{i}=\sum_{\beta} c_{\beta i} \eta_{\beta}
$$

and hence solving the Kohn-Sham equations reduces to solving the following set of secular equations:

${ }^{\otimes}$ Abstract published in Advance ACS Abstracts, December 15, 1994.

$$
\sum_{\beta}\left(F_{\alpha \beta}^{\mathrm{KS}}-\epsilon_{i} S_{\alpha \beta}\right) c_{\beta i}=0
$$

where $\mathrm{F}_{\alpha \beta}^{\mathrm{KS}}$ is the Kohn-Sham matrix and is given by

$\mathbf{F}_{\alpha \beta}^{\mathrm{KS}}=\left\langle\eta_{\alpha}\left|-\frac{1}{2} \nabla^{2}+v(\mathbf{r})+\int \frac{\varrho\left(\mathbf{r}^{\prime}\right)}{\left|\mathbf{r}-\mathbf{r}^{\prime}\right|} \mathrm{d} \mathbf{r}^{\prime}+v_{\mathrm{xc}}(\mathbf{r})\right| \eta_{\beta}\right\rangle$

The first Hohenberg-Kohn theorem ${ }^{1}$ tells us that a functional $E_{x c}[\varrho]$ exists; however, it does not give its functional form, nor does it give any information on how to make systematic improvements to any approximate form of $E_{x c}[Q]$ we might have. The conventional way to proceed is to use the uniform electron gas results for the exchange ${ }^{3}$ and correlation energy ${ }^{4}$ as a starting approximation to $E_{\mathrm{xc}}[\varrho]$, and this constitutes the local density approximation (LDA). In using the LDA we are assuming that the electron distribution in a system is locally homogeneous, and this is clearly not the case for atoms and molecules where the density may be rapidly varying. The local density approximation can be refined by introducing "correction" functionals that depend on both the electron density $\varrho(\mathbf{r})$ and its gradient $\nabla Q(\mathbf{r}) ;{ }^{1}$ this is done in an attempt to introduce information concerning the local inhomogeneity of the system, and there are currently a wealth of gradient correction functionals available in the literature. ${ }^{5-9}$ The uniform electron gas result plus gradient correction approach has proved very successful, and there are today many composite forms for $E_{\mathrm{xc}^{-}}$ $[\varrho]$ available in the literature such as B-LYP; ${ }^{10-12}$ there also exist successful composite forms for $E_{\mathrm{xc}}[\varrho]$ that follow the above approach, but also "mix" in a component of Hartree-Fock exchange. ${ }^{13,14}$

In Kohn-Sham density functional theory it is common practice to partition the exchange-correlation functional into exchange and correlation contributions ${ }^{15}$ such that

$$
E_{\mathrm{xc}}[\varrho]=E_{\mathrm{x}}[\varrho]+E_{\mathrm{c}}[\varrho]
$$

Considering only the exchange contribution to $E_{\mathrm{xc}}[\varrho]$, Becke ${ }^{5}$ proposed augmenting the Dirac (LDA) exchange functional with a gradient correction containing a parameter $\beta$; this parameter was found to be 0.0042 from a least squares fit of the composite exchange functional, 


$$
\begin{gathered}
E_{\mathrm{x}}^{\mathrm{B}}=\int C_{\mathrm{x}} \varrho^{4 / 3}-\beta \sum_{\sigma} \varrho_{\sigma}^{4 / 3} \frac{x_{\sigma}{ }^{2}}{\left(1+6 \beta x_{\sigma} \sinh ^{-1} x_{\sigma}\right)} \mathrm{d} \mathbf{r} \\
x_{\sigma}=\frac{\mid \nabla \varrho_{\sigma}}{\varrho_{\sigma}^{4 / 3}}
\end{gathered}
$$

to the Hartree-Fock exchange energies of the six noble gas atoms. This form for the exchange when coupled with a suitable form for the correlation energy $E_{\mathrm{c}}[\varrho]$, such as the LYP correlation $^{6,7}$ functional, gives significantly better molecular properties than the LDA; this is particularly the case for thermochemical properties. . $^{5,10-12,16-18}$ Using this form for the exchange functional does however lead to bond lengths that are slightly too long and atomization energies that are too large for most molecules; ${ }^{16,17}$ this form of the exchange functional is clearly not perfect, and hence there is room for improvement. $\mathrm{We}^{17}$ recently attempted to improve the performance of B-LYP with some degree of success by making a subtle modification to the form of the B-LYP functional; however, the modified functional was still in the vein of a local density approximation plus gradient-corrected functional approach. This may not be the best way to proceed, and perhaps we should be examining alternative approaches for obtaining the exchange contribution to $E_{\mathrm{xc}}[\varrho]$.

One such approach is the Gaussian function model for the exchange energy first derived by Ghosh and Parr in $1986^{19}$ and later developed by Lee and Parr. ${ }^{20}$ This approach to obtaining the exchange energy is attractive because it does not make any reference to the uniform electron gas model and does not assume that the electron density is locally homogeneous. Further, the expression one arrives at for the exchange energy depends not only on the electron density $\varrho(\mathbf{r})$ but also on higher derivatives. Several workers have made contributions to this area. ${ }^{21-24}$ The approach emanates from the work of Ghosh, Berkowitz, and Parr ${ }^{21}$ who proposed a phase space distribution function $f(\mathbf{r}, \mathbf{p})$ describing a system of $N$ electrons. Ghosh and $\mathrm{Parr}^{19}$ then used this form of $f(\mathbf{r}, \mathbf{p})$ to derive an approximate expression for the exchange energy $K_{\mathrm{G}}$ :

$$
K_{\mathrm{G}}=\frac{\pi}{2} \int \varrho(\mathbf{r})^{2} \beta(\mathbf{r}) \mathrm{d} \mathbf{r}
$$

where $\beta(\mathbf{r})=1 / k T(\mathbf{r})$, and $T(\mathbf{r})$ is a local temperature defined below. They calculated the exchange energies of the six noble gas atoms using $K_{\mathrm{G}}$, and later Lee and Parr ${ }^{20}$ performed a further series of calculations on the noble gas atoms where they used several variants of $K_{\mathrm{G}}$. It was concluded that the Gaussian model for the exchange energy was superior to the local density approximation at modeling the Hartree-Fock exchange energy in atoms. ${ }^{20}$

To the best of our knowledge this general approach to the exchange energy has not been applied to molecules before, and the aim of the present work is to compare its performance with the Hartree-Fock exchange. In this work we have performed a series of calculations using the Gaussian model exchange functional $K_{\mathrm{G}}{ }^{19,20}$ and the related Bessel model exchange functional $K_{\mathrm{T}}{ }^{20}$ on the hydrogen and nitrogen molecules; the resulting potential energy surfaces, equilibrium bond lengths, and exchange energies were compared with those obtained using Hartree-Fock theory. We have also performed further calculations on hydrogen and nitrogen using variants of $K_{\mathrm{G}}$ and $K_{\mathrm{T}}$ which were derived using simple scaling arguments.

\section{Theory}

We shall first summarize the derivation of the Gaussian and Bessel models for the exchange energy functional given by Lee and Parr. ${ }^{20}$ They commenced with the Hartree-Fock equation for the exchange energy $K_{\mathrm{HF}}$ for a system with $N$ electrons, written in terms of the first-order reduced density matrix $\varrho_{1}$ $\left(\mathbf{r}_{1}, \mathbf{r}_{2}\right)$ :

$$
K_{\mathrm{HF}}=\frac{1}{4} \iint \frac{\left|\varrho_{1}\left(\mathbf{r}_{1}, \mathbf{r}_{2}\right)\right|^{2}}{r_{12}} \mathrm{~d} \mathbf{r}_{1} \mathrm{~d} \mathbf{r}_{2}
$$

By writing the first-order reduced density matrix in terms of the Hartree-Fock orbitals,

$$
\varrho_{1}\left(\mathbf{r}_{1}, \mathbf{r}_{2}\right)=\sum_{i}^{N / 2} 2 \psi_{i}\left(\mathbf{r}_{1}\right) \psi_{i}\left(\mathbf{r}_{2}\right)
$$

the following expression for the exchange energy is obtained:

$$
K_{\mathrm{HF}}=\frac{1}{4} \iint \frac{\left|\sum_{i}^{N / 2} 2 \psi_{i}\left(\mathbf{r}_{1}\right) \psi_{i}\left(\mathbf{r}_{2}\right)\right|^{2}}{r_{12}} \mathrm{~d} \mathbf{r}_{1} \mathrm{~d} \mathbf{r}_{2}
$$

It should be noted that the numerator of eq 14 when integrated yields the total number of electrons $N$ in the system such that

$$
2 N=\iint\left|\sum_{i}^{N / 2} 2 \psi_{i}\left(\mathbf{r}_{1}\right) \psi_{i}\left(\mathbf{r}_{2}\right)\right|^{2} \mathrm{~d} \mathbf{r}_{1} \mathrm{~d} \mathbf{r}_{2}
$$

Center of mass $\mathbf{r}$ and relative coordinates $\mathbf{s}$ are introduced such that

$$
\begin{aligned}
& \mathbf{r}_{1}=\mathbf{r}+\mathrm{s} / 2 \\
& \mathbf{r}_{2}=\mathbf{r}-\mathrm{s} / 2
\end{aligned}
$$

and the molecular orbitals $\psi_{i}\left(\mathbf{r}_{1}\right)$ and $\psi_{i}\left(\mathbf{r}_{2}\right)$ are then expanded in $\mathbf{s}$ about $\mathbf{r}$ through second order. Some algebra leads to the following expression for the exchange energy:

$$
K_{s^{2}}=\pi \iint \varrho(\mathbf{r})^{2}\left(1-\frac{s^{2}}{\beta(\mathbf{r})}\right) s \mathrm{~d} s \mathrm{~d} \mathbf{r}
$$

where

$$
\beta(\mathbf{r})=\frac{3}{2} \frac{\varrho(\mathbf{r})}{t(\mathbf{r}, \varrho(\mathbf{r}))}
$$

and where $t(\mathbf{r}, \varrho(\mathbf{r}))$ is given by ${ }^{23}$

$$
t(\mathbf{r}, Q(\mathbf{r}))=-\frac{1}{8} \nabla^{2} \varrho(\mathbf{r})+\sum_{i}^{N / 2}\left|\nabla \psi_{i}(\mathbf{r})\right|^{2}
$$

This expression for the exchange energy is correct through order $s^{2}$. The related expression for the number of electrons is

$$
N_{\mathrm{s}^{2}}=2 \pi \int \varrho^{2} \int\left(1-\frac{s^{2}}{\beta(\mathbf{r})}\right) s^{2} \mathrm{~d} s \mathrm{~d} \mathbf{r}
$$

To proceed further, the integration over $s$ must be made finite, and to do this, a local approximation is introduced. In this work we shall consider the two approximations, first introduced by Lee and Parr: the Gaussian function approximation and the Bessel function approximation.

(A) In the Gaussian approximation the quantity $1-\left(s^{2} / \beta-\right.$ (r)) in the integrand of eq 18 is approximated as a Gaussian function: 


$$
1-\frac{s^{2}}{\beta} \approx \mathrm{e}^{-\left(s^{2} / \beta(\mathbf{r})\right)}
$$

Performing the integration over $s$ gives

$$
K_{\mathrm{G}}=\frac{\pi}{2} \int Q(\mathbf{r})^{2} \beta(\mathbf{r}) \mathrm{d} \mathbf{r}
$$

Likewise, the number of electrons is given by

$$
N_{\mathrm{G}}=\frac{\pi^{3 / 2}}{2} \int \varrho(\mathbf{r})^{2} \beta(\mathbf{r})^{3 / 2} \mathrm{~d} \mathbf{r}
$$

In this work we solve the Kohn-Sham equations, and therefore we require the contribution of $K_{\mathrm{G}}$ to the Kohn-Sham matrix $\mathbf{F}_{\alpha \beta}^{\mathrm{KS}}$. We obtain this contribution, which we denote as $F_{\alpha \beta}^{\mathrm{G}}$, by finding the exchange contribution to the condition that the energy is stationary with respect to the Kohn-Sham canonical orbital coefficients $c_{\beta i}$, subject to the constraint that the orbitals remained normalized. The contribution of $K_{\mathrm{G}}$ to the total Kohn-Sham matrix $\mathbf{F}_{\alpha \beta}^{\mathrm{KS}}$ may then be written as

$$
F_{\alpha \beta}^{\mathrm{G}}=-\frac{3 \pi}{8}(A-B+C+D)
$$

where $A, B, C$, and $D$ are defined as

$$
\begin{aligned}
& A=\left\langle\eta_{\alpha}\left|\frac{12 \varrho^{2}}{t}\right| \eta_{\beta}\right\rangle \\
& B=\left\langle\nabla \eta_{\alpha}\left|\frac{\rho^{3}}{t^{2}}\right| \nabla \eta_{\beta}\right\rangle \\
& C=\left\langle\nabla^{2} \eta_{\alpha}\left|\frac{\rho^{3}}{2 t^{2}}\right| \eta_{\beta}\right\rangle \\
& D=\left\langle\eta_{\alpha}\left|\frac{\rho^{3}}{2 t^{2}}\right| \nabla^{2} \eta_{\beta}\right\rangle
\end{aligned}
$$

Note that there is a minus sign at the front of eq 25 ; this is because we have adopted the usual definition $E_{\mathrm{x}}=-K$. It can further be shown, by performing an integration by parts, that expression 25 for $F_{\alpha \beta}^{G}$ can be written in its more usual form as

$$
\begin{aligned}
F_{\mathrm{a} \beta}^{\mathrm{G}} & =\left\langle\eta_{\alpha}\left|-\frac{\delta K_{\mathrm{G}}}{\delta \varrho(\mathbf{r})}\right| \eta_{\beta}\right\rangle \\
& =\left\langle\eta_{\alpha}\left|v_{\mathrm{x}}^{\mathrm{G}}(\mathbf{r})\right| \eta_{\beta}\right\rangle
\end{aligned}
$$

where $v_{\mathrm{x}}^{\mathrm{G}}(\mathbf{r})$ is the Kohn-Sham exchange potential corresponding to an exchange energy of $-K_{\mathrm{G}}$.

(B) An alternative to the Gaussian function approximation to $1-\left(s^{2} / \beta(\mathbf{r})\right)$ has been suggested by Lee and Parr. ${ }^{20}$ This is the spherical Bessel function approximation,

$$
1-\frac{s^{2}}{\beta(\mathbf{r})} \approx 9\left(\frac{\sin t^{\prime}-t^{\prime} \cos t^{\prime}}{t^{\prime 3}}\right)^{2}
$$

where $t^{\prime}=(5 / \beta)^{1 / 2} s$. The idea behind this approach comes from the derivation of the uniform electron gas exchange result, ${ }^{3}$ where $K=(1 / 4) \iint\left(\left|\varrho_{1}(\mathbf{r}, s)\right|^{2} / s\right) \mathrm{d} \mathbf{r} \mathrm{d} s$, and the expression for the first-order reduced density matrix in center of mass and relative coordinates is

$$
\varrho_{1}(\mathbf{r}, s)=3 \varrho(\mathbf{r})\left(\frac{\sin t^{\prime}-t^{\prime} \cos t^{\prime}}{t^{3}}\right)
$$

with $t^{\prime}=\left(3 \pi^{2} \varrho\right)^{1 / 3} s$. Using the Bessel function approximation, Lee and Parr arrived at the exchange energy $K_{\mathrm{T}}$ where

$$
\begin{aligned}
K_{\mathrm{T}} & =\frac{9 \pi}{20} \int \varrho(\mathbf{r})^{2} \beta(\mathbf{r}) \mathrm{d} \mathbf{r} \\
& =\frac{9}{10} K_{\mathrm{G}}
\end{aligned}
$$

The number of electrons $N_{\Upsilon}$ is then given by the following formula:

$$
N_{\mathrm{T}}=\frac{3 \pi^{2}}{5^{3 / 2}} \int \varrho(\mathbf{r})^{2} \beta(\mathbf{r})^{3 / 2} \mathrm{~d} \mathbf{r}
$$

The contribution of $K_{\mathrm{T}}$ to the Kohn-Sham matrix $\mathrm{F}_{\alpha \beta}^{\mathrm{KS}}$ may be determined as in (A) above. It may then be written as

$$
F_{\alpha \beta}^{\mathrm{T}}=-\frac{27 \pi}{80}(A-B+C+D)
$$

where $A, B, C$, and $D$ are given by eqs 26-29. Again the contribution $F_{\alpha \beta}^{\mathrm{T}}$ to the Kohn-Sham matrix can be written in the form

$$
\begin{aligned}
F_{\alpha \beta}^{\mathrm{T}} & =\left\langle\eta_{\alpha}\left|-\frac{\delta K_{\mathrm{T}}}{\delta \varrho(\mathbf{r})}\right| \eta_{\beta}\right\rangle \\
& =\left\langle\eta_{\alpha}\left|\nu_{\mathbf{x}}^{\mathrm{T}}(\mathbf{r})\right| \eta_{\beta}\right\rangle
\end{aligned}
$$

where $v_{x}^{\mathrm{T}}(\mathbf{r})$ is the Kohn-Sham exchange potential corresponding to an exchange energy of $-K_{\mathrm{T}}$.

2.1. Scaling $\boldsymbol{K}_{\mathrm{G}}$ and $\boldsymbol{K}_{\mathrm{T}}$. In practice we find that both the Gaussian function and Bessel function approximations underestimate the magnitude of the Hartree-Fock exchange energy and the number of electrons in the system such that

$$
\begin{gathered}
K_{\mathrm{HF}}>K_{\mathrm{G}}>K_{\mathrm{T}} \\
N>N_{\mathrm{G}}>N_{\mathrm{T}}
\end{gathered}
$$

We therefore propose a simple scaling of the functionals $K_{\mathrm{G}}$ and $K_{\mathrm{T}}$ to arrive at two new scaled functionals $K_{\mathrm{G}}^{\text {Scaled }}$ and $K_{\mathrm{T}}^{\text {Scaled }}$ where

$$
\begin{aligned}
& K_{\mathrm{G}}^{\text {scaled }}=\frac{N}{N_{\mathrm{G}}} K_{\mathrm{G}} \\
& K_{\mathrm{T}}^{\text {Scaled }}=\frac{N}{N_{\mathrm{T}}} K_{\mathrm{T}}
\end{aligned}
$$

We have also studied this scaled functional in a self-consistent manner. The contribution of $K_{\mathrm{G}}^{\text {Scaled }}$ to the Kohn-Sham matrix $\mathbf{F}_{\alpha \beta}^{\mathrm{KS}}$ is

$$
\begin{aligned}
& =C_{1}^{G}(A-B+C+D)+C_{2}^{G}(E-F+G+H) \\
& =\left\langle\eta_{\alpha}\left|-\frac{\delta K_{\mathrm{G}}^{\text {Scaled }}}{\delta \varrho(\mathbf{r})}\right| \eta_{\beta}\right\rangle \\
& =\left\langle\eta_{\alpha}\left|\nu_{x}(\mathbf{r})\right| \eta_{\beta}\right\rangle
\end{aligned}
$$

where $A, B, C$, and $D$ are given by eqs $26-29$ and 


$$
\begin{gathered}
C_{1}^{\mathrm{G}}=-\frac{3 \pi}{8} \frac{N}{N_{\mathrm{G}}} \\
C_{2}^{\mathrm{G}}=\frac{\pi^{3 / 2}}{4}\left(\frac{3}{2}\right)^{3 / 2} \frac{N K_{\mathrm{G}}}{N_{\mathrm{G}}{ }^{2}} \\
E=\left\langle\eta_{\alpha}\left|\frac{14 \varrho^{5 / 2}}{t^{3 / 2}}\right| \eta_{\beta}\right\rangle \\
F=\left\langle\nabla \eta_{\alpha}\left|\frac{3}{2} \frac{\rho^{7 / 2}}{t^{5 / 2}}\right| \nabla \eta_{\beta}\right\rangle \\
G=\left\langle\nabla \eta_{\alpha}\left|\frac{3}{4} \frac{\rho^{7 / 2}}{t^{5 / 2}}\right| \eta_{\beta}\right\rangle \\
H=\left\langle\nabla \eta_{\alpha}\left|\frac{3}{4} \frac{e^{7 / 2}}{t^{5 / 2}}\right| \nabla^{2} \eta_{\beta}\right\rangle
\end{gathered}
$$

The corresponding contribution to the Kohn-Sham matrix $\mathrm{F}_{\alpha \beta}^{\mathrm{KS}}$ for $K_{\mathrm{T}}^{\mathrm{Scaled}}$ can be obtained using eq 44 by replacing the coefficients $C_{1}^{G}$ and $C_{2}^{G}$ with $C_{1}^{T}$ and $C_{2}^{T}$, respectively, where these are given by

$$
\begin{gathered}
C_{1}^{\mathrm{T}}=-\frac{27 \pi}{80} \frac{N}{N_{\mathrm{T}}} \\
C_{2}^{\mathrm{T}}=\frac{3 \pi^{2}}{2}\left(\frac{3}{10}\right)^{3 / 2} \frac{N K_{\mathrm{T}}}{N_{\mathrm{T}}^{2}}
\end{gathered}
$$

\section{Details of Calculations}

All the calculations reported in this work were performed using the CADPAC computational chemistry code, ${ }^{25}$ which uses regular Gaussian basis sets and has recently been extended to include a Kohn-Sham DFT capability. The version of CADPAC used for this work evaluates all the one-electron and Coulombic integrals analytically. The DFT-related integrals are evaluated by quadrature, full details of which are given elsewhere. ${ }^{26}$ All the new integrals that arise in this paper may be evaluated using the same one-electron three-dimensional quadrature scheme, and they are programmed exactly as written in eqs 23-54. In the course of our calculations we noticed that the quantity $t(\mathbf{r}, \varrho(\mathbf{r}))$ became negative in some instances when using the scaled functionals $K_{\mathrm{G}}^{\text {Scaled }}$ and $K_{\mathrm{T}}^{\text {Scaled }}$. In no cases, however, did we observe a divergence in the energy. We have optimized bond lengths and calculated potential energy surfaces for the hydrogen and nitrogen molecules using a variety of forms for the exchange energy. For $\mathrm{H}_{2}$ we used Dunning DZ basis ${ }^{27}$ augmented with a p-type basis function with exponent 0.8 ; the standard $\mathrm{p}$-function exponent for the $\mathrm{H}_{2} \mathrm{DZP}$ basis is 1.0 ; however, we were unable to converge the KohnSham equations with the new functionals using the standard basis set. For $\mathrm{N}_{2}$ a standard DZP basis ${ }^{27}$ was used. Besides presenting calculations of the self-consistent Kohn-Sham form, we also present "hybrid" DFT calculations.

In a "hybrid" calculation, the Hartree-Fock (SCF) density

\begin{tabular}{|c|c|c|c|}
\hline method & $r_{\mathrm{e}} / \AA$ & $E_{\text {Total }} /$ hartrees & $-E_{\mathrm{x}} /$ hartrees \\
\hline$K_{\mathrm{HF}}$ & 0.735 & -1.13116 & 0.66458 \\
\hline $\begin{array}{l}K_{\mathrm{LDA}} \\
K_{\mathrm{G}} \\
K_{\mathrm{T}} \\
K_{\mathrm{G}}^{\text {Scaled }} \\
K_{\mathrm{T}}^{\text {Scaled }} \\
K_{\mathrm{LDA}+\mathrm{B} 88} \\
K_{\mathrm{K}_{\mathrm{G}}+\mathrm{B} 88} \\
K_{\mathrm{K}_{\mathrm{T}}+\mathrm{B} 88}\end{array}$ & $\begin{array}{l}0.781 \\
0.787 \\
0.817 \\
0.691 \\
0.703 \\
0.754 \\
0.767 \\
0.759\end{array}$ & $\begin{array}{l}\text { hn-Sham } \\
-1.04043 \\
-1.09363 \\
-1.03460 \\
-1.16298 \\
-1.12435 \\
-1.12784 \\
-1.13712 \\
-1.16096\end{array}$ & $\begin{array}{l}0.56039 \\
0.59779 \\
0.52532 \\
0.72326 \\
0.67377 \\
0.56921 \\
0.65490 \\
0.68701\end{array}$ \\
\hline $\begin{array}{l}K_{\mathrm{G}} \\
K_{\mathrm{T}} \\
K_{\mathrm{G}}^{S_{\text {caled }}} \\
K_{\mathrm{T}}^{S_{\text {caled }}}\end{array}$ & $\begin{array}{l}0.771 \\
0.794 \\
0.687 \\
0.701\end{array}$ & $\begin{array}{l}\text { Hybrid } \\
\qquad-1.09164 \\
-1.03061 \\
-1.16132 \\
-1.12307\end{array}$ & $\begin{array}{l}0.61345 \\
0.54637 \\
0.71362 \\
0.66957\end{array}$ \\
\hline
\end{tabular}
is used to calculate the total energy such that

$$
E_{\text {Total }}^{\text {hybrid }}=E_{\text {Total }}^{\mathrm{HF}}-E_{\mathrm{x}}^{\mathrm{HF}}+E_{\mathrm{x}}^{\mathrm{DFT}}\left(\varrho^{\mathrm{HF}}\right)
$$

where $E_{\mathrm{x}}^{\mathrm{HF}}$ is the Hartree-Fock exchange energy and $E_{\mathrm{x}}^{\mathrm{DFT}}$ is the corresponding DFT expression for the exchange energy. We
TABLE 1: Equilibrium Bond Lengths, Total Energies, and Exchange Energies for $\mathrm{H}_{2}$ with a DZP Basis

have also adopted the following convention throughout this work,

$$
K=-E_{\mathrm{x}}
$$

and as a result $K_{\mathrm{G}}$ and $K_{\mathrm{T}}$ are positive and all exchange energies presented in this paper are positive numbers.

\section{Results of the Calculations}

4.1. The Hydrogen Molecule. Table 1 reports calculations for optimized equilibrium bond lengths, total energies, and exchange energies for the $\mathrm{H}_{2}$ molecule using all of the methods described in sections 2 and 3 . Figures $1-3$ show the potential energy curves for $0 \leq R \leq 2 \AA$ for these methods. The figures plotted are on absolute energy scales.

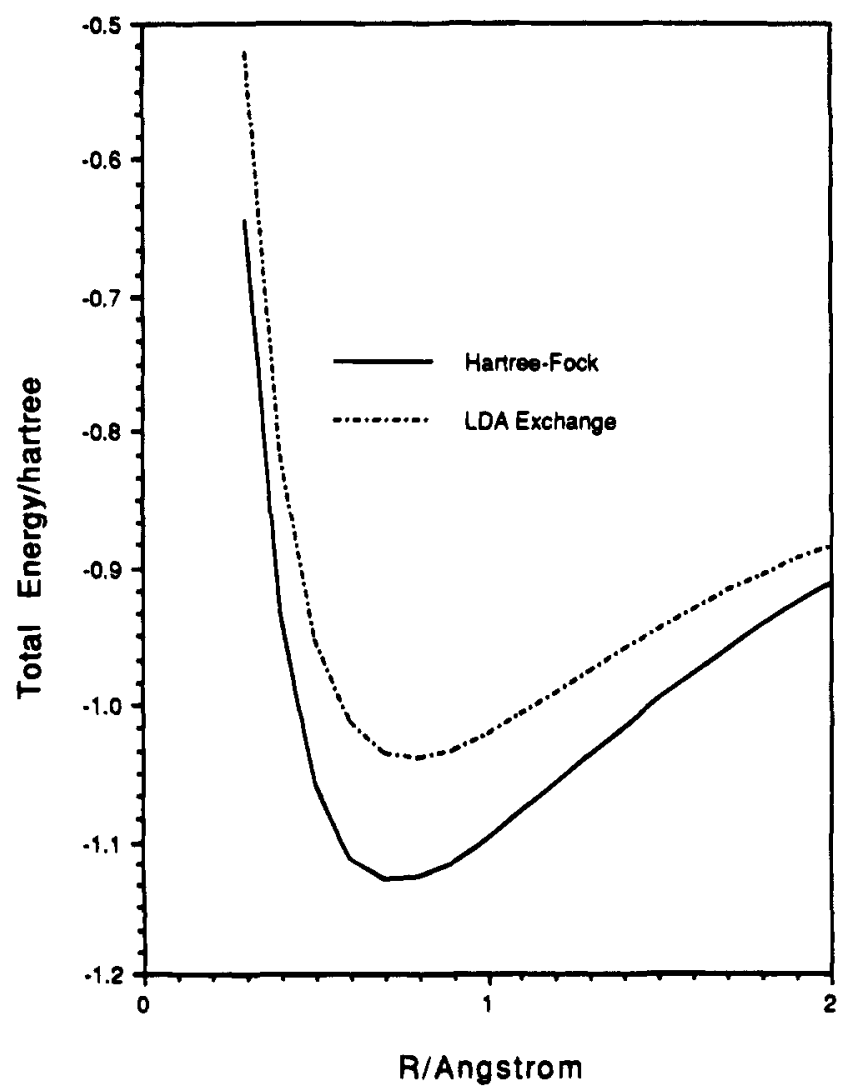

Figure 1. Hydrogen potential energy curves using the HF and KohnSham LDA forms for exchange with a DZP basis. 


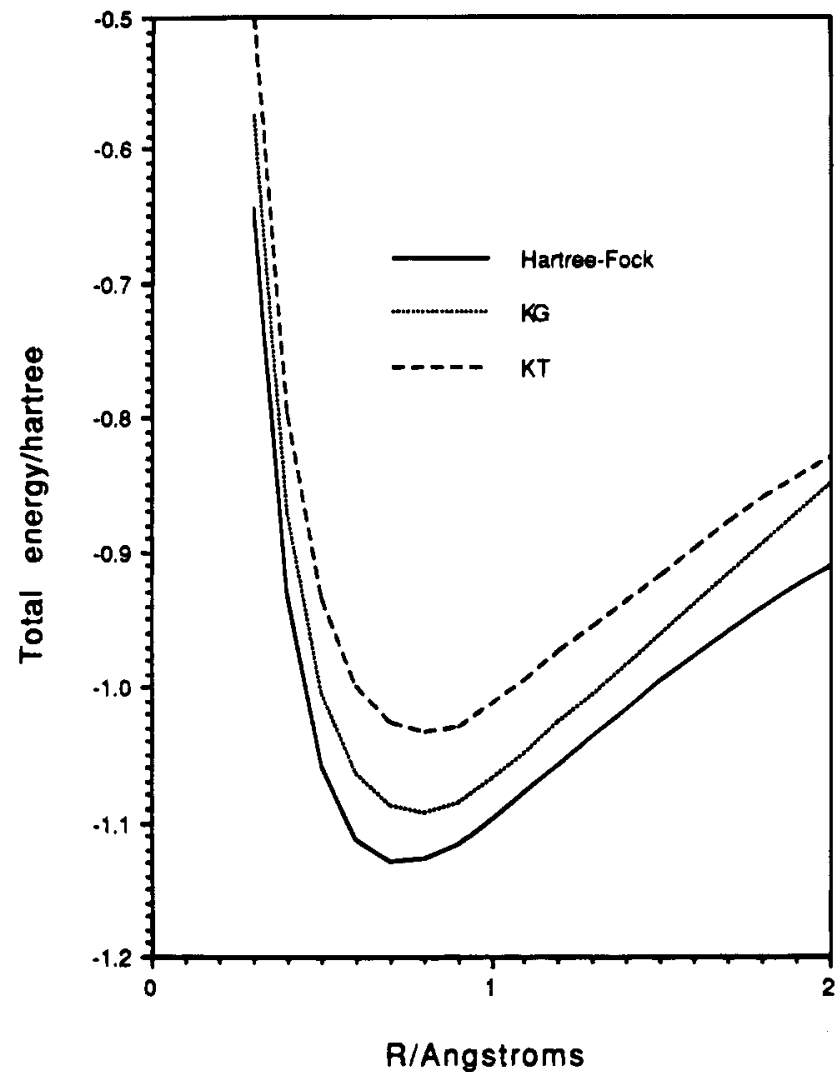

Figure 2. Hydrogen potential energy curves using the HF and KohnSham $K_{\mathrm{G}}$ and $K_{\mathrm{T}}$ forms for exchange and a DZP basis set.

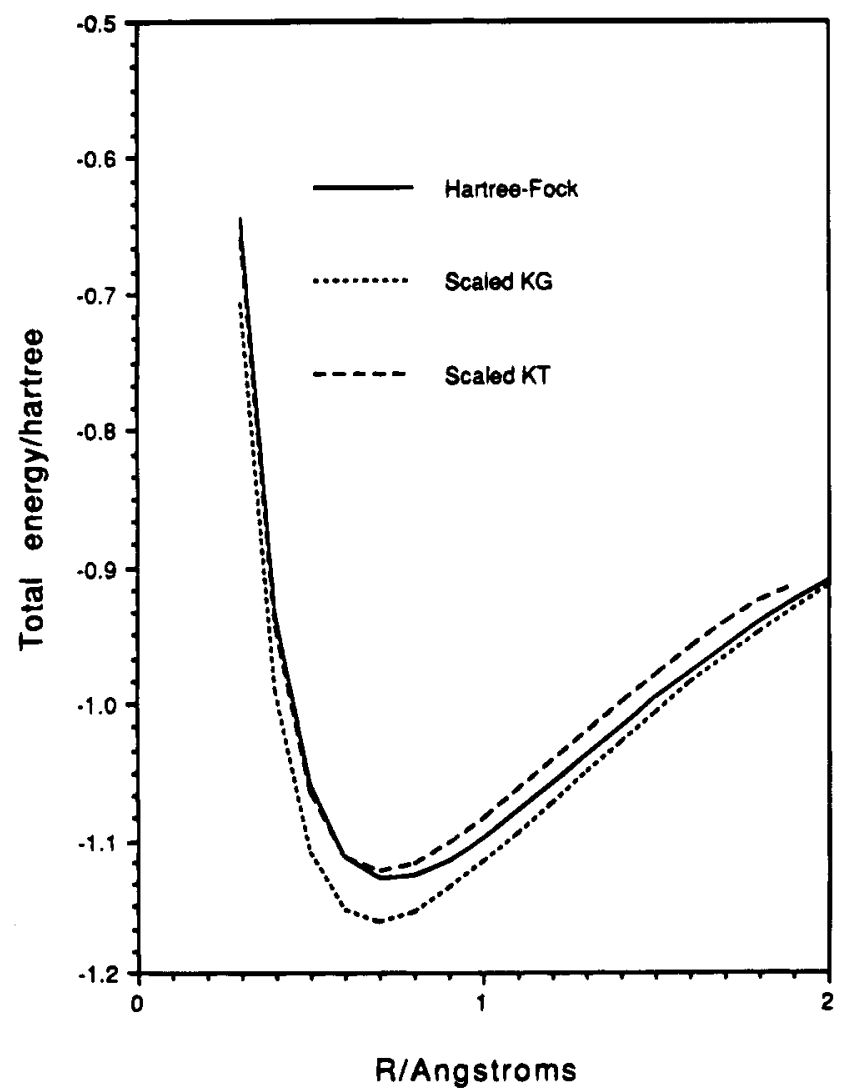

Figure 3. Hydrogen potential energy curves using the HF and KohnSham Scaled $K_{\mathrm{G}}$ and $K_{\mathrm{T}}$ forms for the exchange with a DZP basis set.

Because the theory of this paper uses approximations to the Hartree-Fock exchange energy, we shall be comparing our results to the corresponding Hartree-Fock results. Thus, a
TABLE 2: Equilibrium Bond Lengths, Total Energies, and Exchange Energies for $\mathrm{N}_{2}$ with a DZP Basis for Hartree-Fock and Calculations Using the LDA, $K_{\mathrm{G}}$, and $K_{\mathrm{T}}$ Forms for the Exchange

\begin{tabular}{|c|c|c|c|}
\hline method & $r_{\mathrm{e}} / \AA \AA$ & $E_{\text {Total }} /$ hartrees & $-E_{\mathrm{x}} /$ hartrees \\
\hline$K_{\mathrm{HF}}$ & 1.083 & -108.95896 & 13.12762 \\
\hline $\begin{array}{l}K_{\mathrm{LDA}} \\
K_{\mathrm{G}} \\
K_{\mathrm{T}} \\
K_{\mathrm{LDA}+\mathrm{B} 88} \\
K_{\mathrm{K}_{\mathrm{G}}+\mathrm{B} 88} \\
K_{\mathrm{K}_{\mathrm{T}}+\mathrm{B} 88}\end{array}$ & $\begin{array}{l}1.131 \\
1.051 \\
1.074 \\
1.138 \\
1.055 \\
1.075\end{array}$ & $\begin{array}{l}\text { hn-Sham } \\
-107.70761 \\
-108.13014 \\
-106.90877 \\
-109.03958 \\
-108.73267 \\
-109.00497\end{array}$ & $\begin{array}{l}11.91700 \\
12.27315 \\
10.93974 \\
13.14942 \\
12.90077 \\
13.13729\end{array}$ \\
\hline $\begin{array}{l}K_{\mathrm{G}} \\
K_{\mathrm{T}}\end{array}$ & $\begin{array}{l}1.050 \\
1.074\end{array}$ & $\begin{array}{l}\text { Hybrid } \\
-108.11357 \\
-106.88353\end{array}$ & $\begin{array}{l}12.33910 \\
11.06660\end{array}$ \\
\hline
\end{tabular}

comparison of Figures 1 and 2 shows that the Kohn-Sham $K_{\mathrm{T}}$ is inferior to the Kohn-Sham LDA curve, whereas the KohnSham $K_{\mathrm{G}}$ curve is an improvement in the vicinity of the equilibrium bond length. Figure 3 shows that the Kohn-Sham scaled $K_{\mathrm{G}}$ and $K_{\mathrm{T}}$ curves are an improvement over the unscaled $K_{\mathrm{G}}$ and $K_{\mathrm{T}}$ curves. These conclusions are further demonstrated in Table 1, where it is observed that

(i) for bond lengths the Kohn-Sham results are

$$
K_{\mathrm{G}}^{\text {Scaled }}<K_{\mathrm{T}}^{\text {scaled }}<K_{\mathrm{HF}}<K_{\mathrm{LDA}}<K_{\mathrm{G}}<K_{\mathrm{T}}
$$

(ii) for the exchange energies the Kohn-Sham results are

$$
K_{\mathrm{G}}^{\text {scaled }}>K_{\mathrm{T}}^{\text {scaled }}>K_{\mathrm{HF}}>K_{\mathrm{G}}>K_{\mathrm{LDA}}>K_{\mathrm{T}}
$$

One might say that $K_{\mathrm{G}}$ and $K_{\mathrm{LDA}}$ give similar results, but that $K_{\mathrm{T}}$ is inferior. The scaled results are rather better compared to the unscaled, for the bond lengths; the differences for $K_{\mathrm{G}}$ $\left(K_{\mathrm{G}}^{\text {scaled }}\right)$ are $+0.052(-0.044)$, and for $K_{\mathrm{T}}\left(K_{\mathrm{T}}^{\text {scaled }}\right)$ they are $+0.082(-0.032)$.

As far as $\mathrm{H}_{2}$ is concerned, $K_{\mathrm{G}}, K_{\mathrm{G}}^{\text {scaled }}$, and $K_{\mathrm{T}}^{\text {scaled }}$ offer an improvement over LDA, when compared to the Hartree-Fock results. The "hybrid" results for the scaled $K_{\mathrm{G}}$ and $K_{\mathrm{T}}$ are very close to the Kohn-Sham results. We found that the use of Hartree-Fock densities in the scaled functionals, i.e. the "hybrid" scheme, gave virtually identical potential energy curves, not presented, to the Kohn-Sham scaled calculations.

We compute for the Kohn-Sham/hybrid schemes that $N_{\mathrm{G}}=$ $1.85058 / 1.81075$ and $N_{\mathrm{T}}=1.53004 / 1.47497$. The trend $N_{\mathrm{T}}$ $<N_{\mathrm{G}}<N$ is consistent with the earlier findings of Lee and Parr for atoms. ${ }^{20}$

That the "hybrid" results are very close to the Kohn-Sham results may be important because we encountered considerable convergence difficulties with the Kohn-Sham calculations for each of these new exchange functionals. For example with Dunning's TZ2P basis ${ }^{28}$ we were unable to converge satisfactorily any of the $K_{\mathrm{G}}, K_{\mathrm{T}}, K_{\mathrm{G}}^{\text {Scaled }}$, and $K_{\mathrm{T}}^{\text {scaled }}$ self-consistent Kohn-Sham calculations. We also experienced difficulties converging the Kohn-Sham equations with Dunning's standard DZP basis; ${ }^{27}$ however; this problem was cured by the use of the smaller (more diffuse) p-function exponent of 0.8 instead of the standard value of 1.0 .

4.2. The Nitrogen Molecule. Table 2 reports calculations for equilibrium bond lengths, total energies, and exchange energies for the $\mathrm{N}_{2}$ molecule using some of the methods described in sections 2 and 3 . Figures $4-6$ show the potential energy curves for $0 \leq R \leq 2 \AA$, and all the figures presented are plotted on absolute energy scales. 


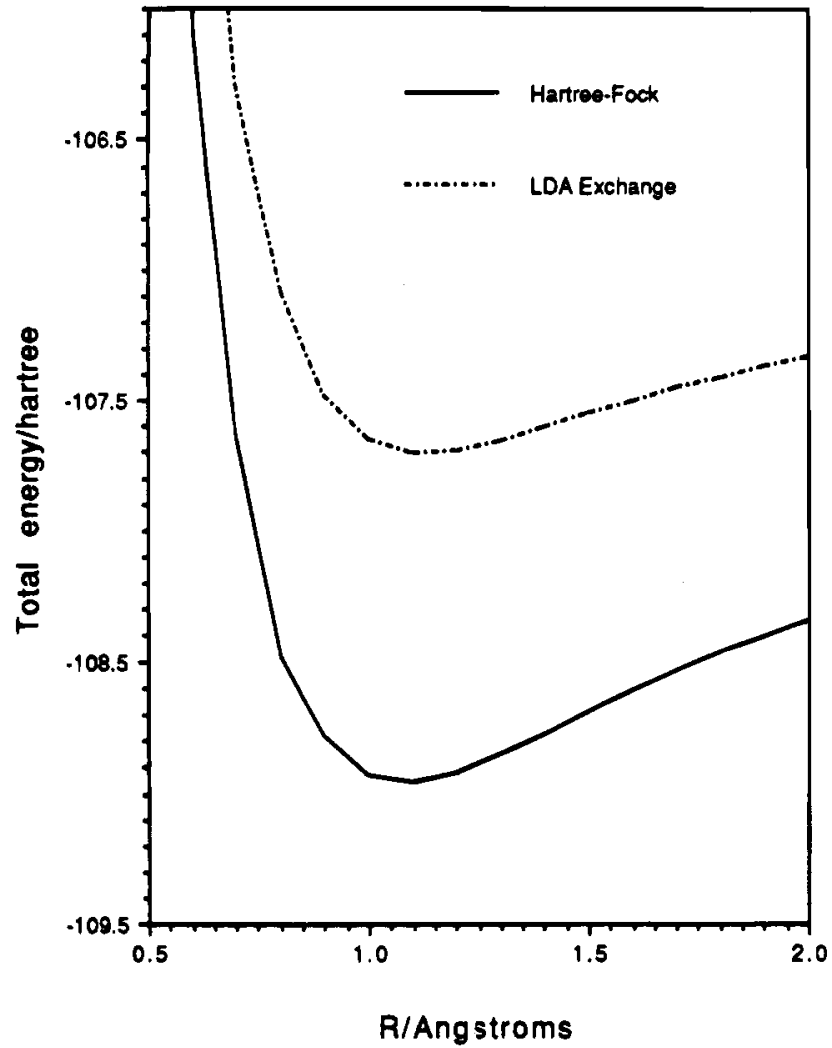

Figure 4. Nitrogen potential energy curves using the HF and KohnSham methods with a DZP basis set.

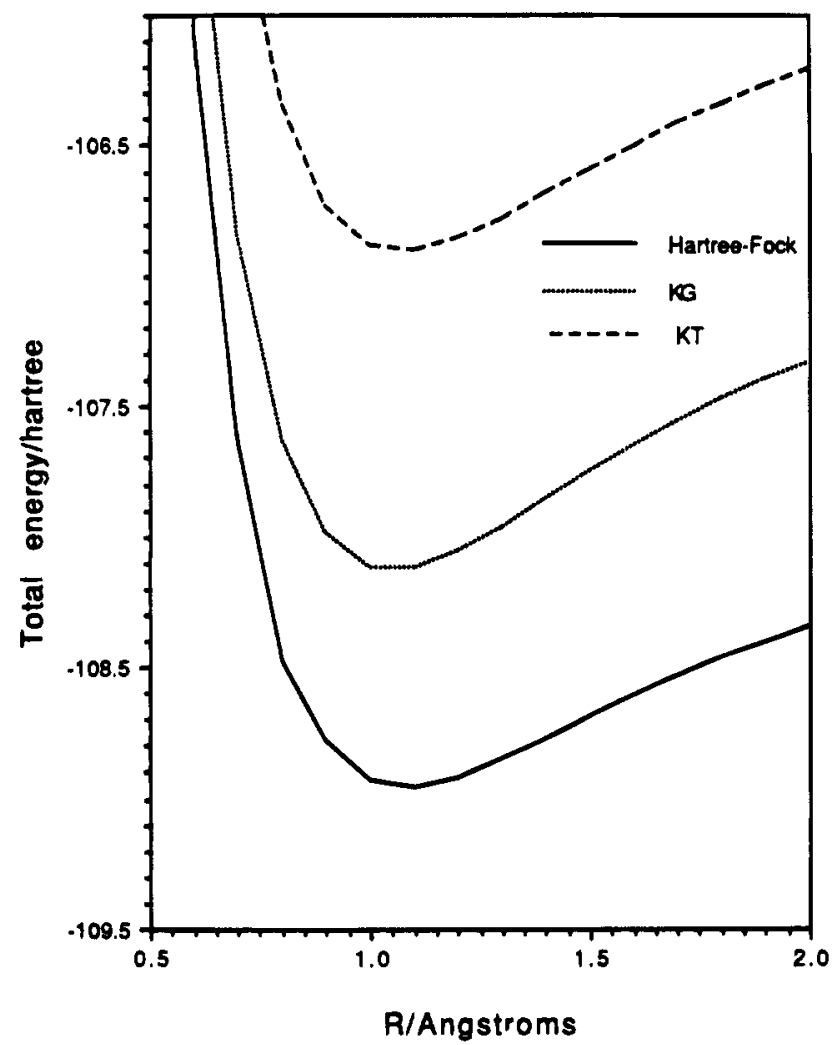

Figure 5. Nitrogen potential energy curves using the HF and KohnSham $K_{\mathrm{G}}$ and $K_{\mathrm{T}}$ forms for exchange with a DZP basis set.

As with $\mathrm{H}_{2}$, we compare the results of our calculations using $K_{\mathrm{G}}$ and $K_{\mathrm{T}}$ with the corresponding Hartree-Fock results. We see from a comparison of Figures 4 and 5 that, as with $\mathrm{H}_{2}$, the Kohn-Sham $K_{\mathrm{T}}$ potential energy curve is inferior to the KohnSham LDA curve, whereas the Kohn-Sham $K_{\mathrm{G}}$ curve is an

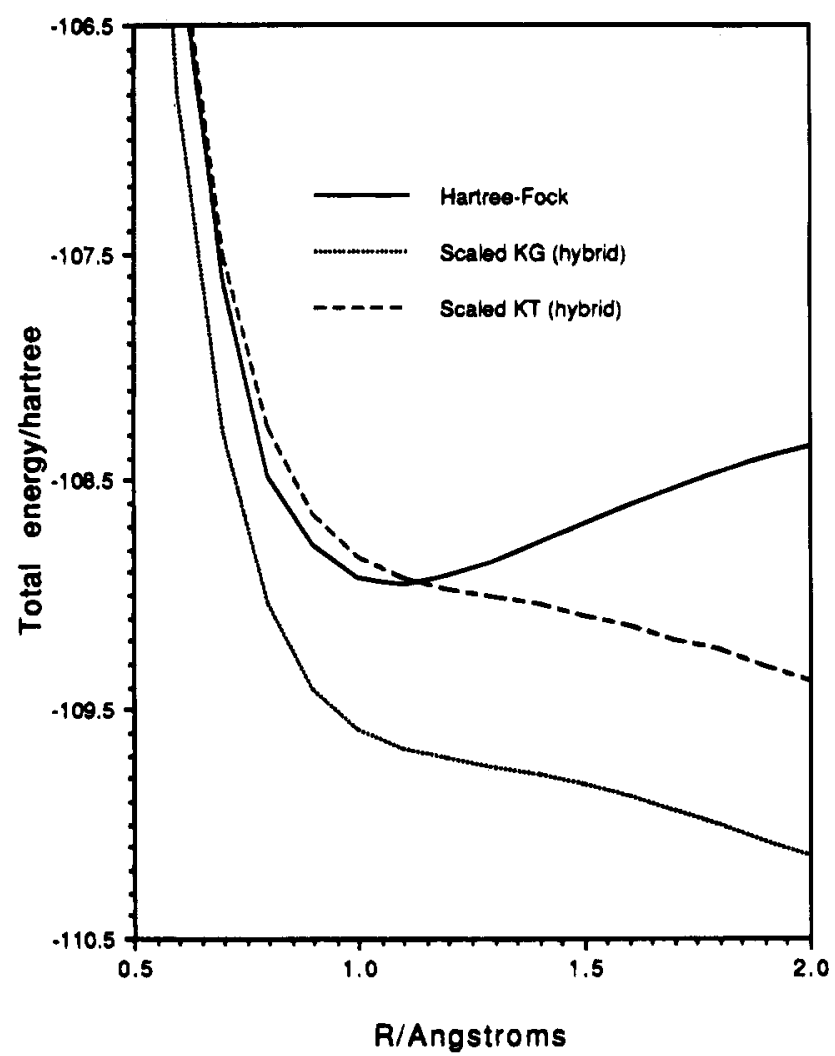

Figure 6. Nitrogen potential energy curves for HF and hybrid calculations with the scaled $K_{\mathrm{G}}$ and $K_{\mathrm{T}}$ exchange functionals with a DZP basis set.

improvement in the vicinity of the equilibrium bond length. These conclusions are further demonstrated in Table 2, where it is observed that

(i) for bond lengths the Kohn-Sham results are

$$
K_{\mathrm{G}}<K_{\mathrm{T}}<K_{\mathrm{HF}}<K_{\mathrm{LDA}}
$$

indicating that, in contrast to our findings for $\mathrm{H}_{2}, K_{\mathrm{G}}$ and $K_{\mathrm{T}}$ predict bond lengths that are shorter than the Hartree-Fock bond length for $\mathrm{N}_{2}$. This is significant because the Hartree-Fock approach is well-known to underestimate bond lengths.

(ii) We observe the following trends for the Kohn-Sham exchange energies:

$$
K_{\mathrm{HF}}>K_{\mathrm{G}}>K_{\mathrm{LDA}}>K_{\mathrm{T}}
$$

which is consistent with our earlier findings for $\mathrm{H}_{2}$.

The effective number of electrons in the exchange for all these methods is less than $14\left(N_{\mathrm{G}}=12.54322 / 12.45807\right.$ and $N_{\mathrm{T}}=$ $10.18501 / 10.09691$, such that $N_{\mathrm{T}}<N_{\mathrm{G}}<N$; these findings are consistent with our earlier results for $\mathrm{H}_{2}$ and with those of Lee and Parr for atoms. ${ }^{20}$

Note the absence of results for the scaled functionals $K_{\mathrm{G}}^{\text {Scaled }}$ and $K_{\mathrm{T}}^{\text {Scaled }}$ for both Kohn-Sham and "hybrid" methods in Table 2. We were unable to converge the Kohn-Sham equations for these functionals with either the DZP or TZ2P basis sets. Figure 6 shows the "hybrid" DFT potential energy curves for $K_{\mathrm{G}}^{\text {scaled }}$ and $K_{\mathrm{T}}^{\text {scaled }}$ with a DZP basis; we note that both these functionals have no observable minima. Because, for a given basis set, we find that there is very good agreement between the Kohn-Sham and "hybrid" results, we conclude that the Kohn-Sham potential energy curves for nitrogen with $K_{\mathrm{G}}^{\text {Scaled }}$ and $K_{\mathrm{T}}^{\text {Scaled }}$ are likely to display behavior similar to that in Figure 6. We therefore cannot recommend the further use of the scaled functionals $K_{\mathrm{G}}^{\mathrm{Scaled}}$ and $K_{\mathrm{T}}^{\mathrm{Scaled}}$. 
TABLE 3: Exchange Energies/hartrees Predicted for the Six Noble Gas Atoms Using Different Forms for the Exchange Energy with Becke's 1988 Gradient Correction ${ }^{\circ}$

\begin{tabular}{lrrrrl}
\hline & & $E_{\mathrm{x}}^{\mathrm{B}} \beta=$ & $E_{\mathrm{x}}^{\mathrm{G}} \beta=$ & \multicolumn{1}{c}{$E_{\mathrm{x}}^{\mathrm{T}} \beta=$} & \\
atom & \multicolumn{1}{c}{$E_{\mathrm{x}}^{\mathrm{HF}}$} & \multicolumn{1}{c}{0.0042} & \multicolumn{1}{c}{0.0015} & \multicolumn{1}{c}{0.0090} & \multicolumn{1}{c}{ basis } \\
\hline $\mathrm{He}$ & -1.026 & -1.025 & -0.982 & -1.033 & $13 \mathrm{~s}$ \\
$\mathrm{Ne}$ & -12.115 & -12.140 & -12.075 & -12.208 & $\mathrm{TZ2P}$ \\
$\mathrm{Ar}$ & -30.181 & -30.149 & -30.236 & -30.136 & $631 \mathrm{G}^{*}$ \\
$\mathrm{Kr}$ & -93.837 & -93.816 & -95.957 & -93.520 & $4 \mathrm{~s} 3 \mathrm{p}^{2 \mathrm{~d}}$ \\
$\mathrm{Xe}$ & -179.002 & -178.978 & -185.112 & -178.545 & $10 \mathrm{~s} 8 \mathrm{p} 6 \mathrm{~d}$ \\
$\mathrm{Rn}$ & -387.205 & -387.215 & -405.183 & -385.826 & $6 \mathrm{~s} 5 \mathrm{p} 3 \mathrm{~d} 1 \mathrm{f}$
\end{tabular}

${ }^{a}$ All the calculations performed were of the "hybrid" type.

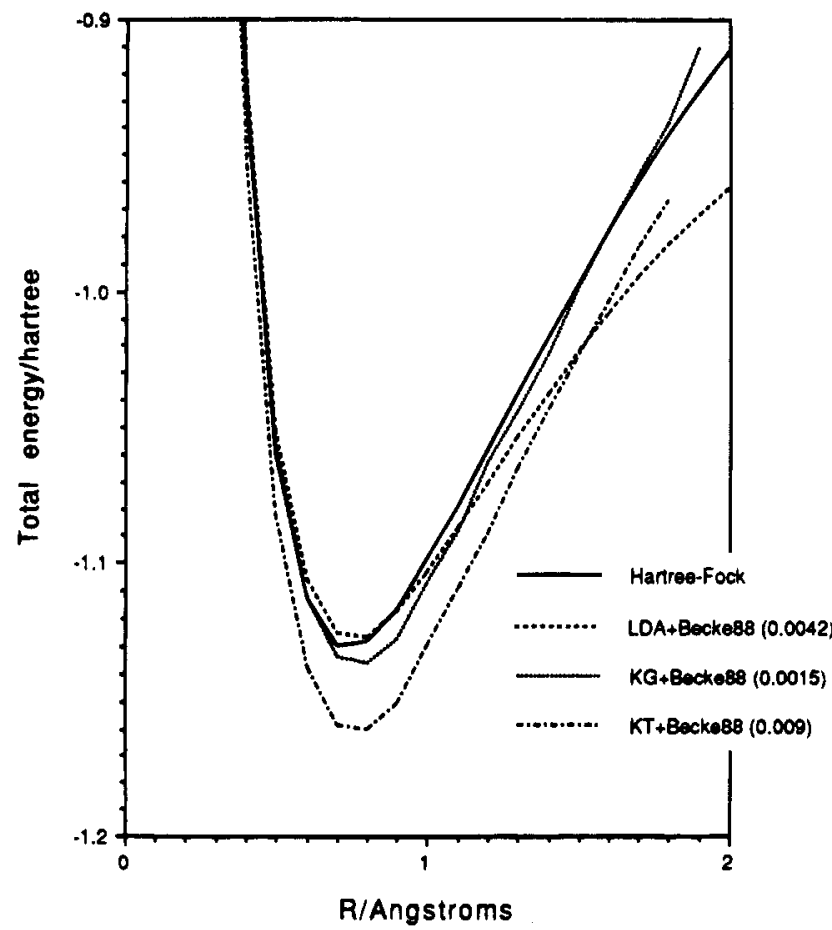

Figure 7. Hydrogen potential energy curves for $\mathrm{HF}$ and Kohn-Sham calculations with LDA, $K_{\mathrm{G}}$, and $K_{\mathrm{T}}$ with Becke's 1988 correction using a DZP basis set.

4.3. Correcting the Asymptotic Behavior of $K_{\mathrm{G}}$ and $K_{\mathrm{T}}$. The Hartree-Fock exchange energy expression of eq 12 may be written as

$$
K_{\mathrm{HF}}=\int \varrho\left(\mathbf{r}_{1}\right) \lambda_{\mathrm{x}} \mathrm{d} \mathbf{r}_{1}
$$

where the quantity $\lambda_{x}$ is given by

$$
\lambda_{\mathrm{x}}=\frac{1}{4 \varrho\left(\mathbf{r}_{1}\right)} \int \frac{\left|\varrho_{1}\left(\mathbf{r}_{1}, \mathbf{r}_{2}\right)\right|^{2}}{r_{12}} \mathrm{~d} \mathbf{r}_{2}
$$

Taking the asymptotic limit of eq $62, r_{1} \rightarrow \infty$, we observe the correct asymptotic limit for $\lambda_{\mathrm{x}}$ is the following:

$$
\lambda_{\mathrm{x}} \rightarrow \frac{1}{2 r_{1}}
$$

In this limit the electron density and Kohn-Sham orbitals have the following behavior:

$$
\begin{gathered}
\varrho(\mathbf{r}) \sim \mathrm{e}^{-\alpha r} \\
\psi_{i}(\mathbf{r}) \sim \mathrm{e}^{-(\alpha / 2) r}
\end{gathered}
$$

Both $K_{\mathrm{G}}$ and $K_{\mathrm{T}}$ can be written in the form

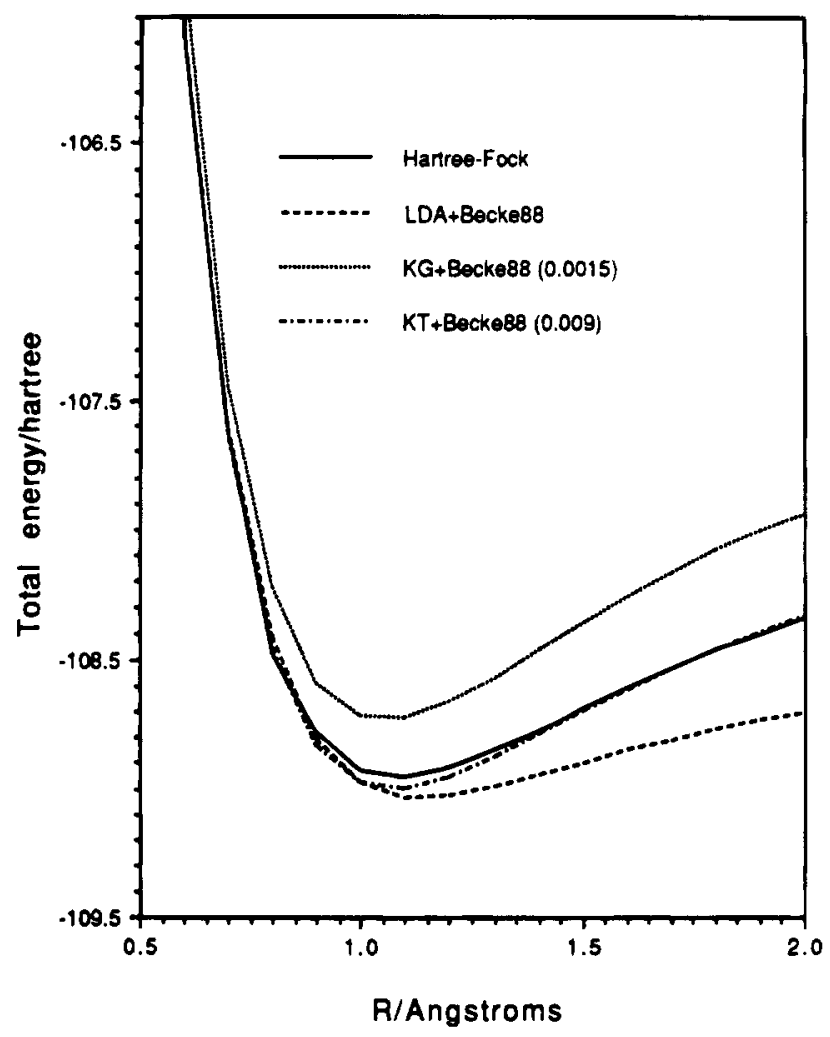

Figure 8. Nitrogen potential energy curves for HF and Kohn-Sham calculations using the LDA, $K_{\mathrm{G}}$, and $K_{\mathrm{T}}$ forms for exchange with Becke's 1988 correction for a DZP basis set.

$$
K=C \int \frac{\varrho^{3}(\mathbf{r})}{\frac{1}{2} \sum_{i}^{N / 2}\left(\left|\nabla \psi_{i}(\mathbf{r})\right|^{2}-\psi_{i}(\mathbf{r}) \nabla^{2} \psi_{i}(\mathbf{r})\right)} \mathrm{dr}
$$

where $C$ is a constant. $\lambda_{\mathrm{x}}$ for $K_{\mathrm{G}}$ and $K_{\mathrm{T}}$ is of the general form

$$
\lambda_{\mathrm{x}}=C \frac{\varrho^{2}\left(\mathbf{r}_{\mathbf{1}}\right)}{\frac{1}{2} \sum_{i}^{N / 2}\left(\left|\nabla \psi_{i}\left(\mathbf{r}_{1}\right)\right|^{2}-\psi_{i}\left(\mathbf{r}_{1}\right) \nabla^{2} \psi_{i}\left(\mathbf{r}_{\mathbf{1}}\right)\right)}
$$

On substituting eqs 64 and 65 into 67 , we find that $\lambda_{x}$ does not possess the correct asymptotic behavior of eq 63 for $K_{\mathrm{G}}$ and $K_{\mathrm{T}}$. We have attempted to correct for this problem by augmenting $K_{\mathrm{G}}$ and $K_{\mathrm{T}}$ with Becke's 1988 gradient correction, ${ }^{5}$ which was designed to correct for the Dirac $C_{\mathrm{x}} \int \varrho^{4 / 3}(\mathbf{r}) \mathrm{dr}$ also having the incorrect asymptotic behavior. The gradient correction has the following functional form:

$E_{\mathrm{x}}^{\text {Becke } 1988}[\varrho]=-\sum_{\sigma} \int \beta \varrho_{\sigma}^{4 / 3} \frac{x_{\sigma}{ }^{2}}{\left(1+6 \beta x_{\sigma} \sinh ^{-1} x_{\sigma}\right)} \mathrm{d} \mathbf{r}$

where

$$
x_{\sigma}=\frac{\left|\nabla \varrho_{\sigma}\right|}{\varrho_{\sigma}^{4 / 3}}
$$

and $\sigma$ corresponds to spin "up" or spin "down".

The parameter $\beta$ in the original prescription ${ }^{5}$ was found to be 0.0042 , obtained by fitting the LDA exchange plus the Becke 
TABLE 4: Equilibrium Bond Lengths for $\mathrm{H}_{2}, \mathbf{N}_{2}, \mathrm{HF}, \mathrm{CO}$, and $\mathrm{F}_{2}$, with Different Forms of the Exchange-Correlation Functional $E_{\text {xc }}[\varrho]$ using DZP and TZ2P Basis Sets

\begin{tabular}{|c|c|c|c|c|c|c|c|c|c|c|}
\hline \multirow[b]{2}{*}{ method } & \multicolumn{2}{|c|}{$\mathrm{H}_{2}$} & \multicolumn{2}{|c|}{$\mathrm{N}_{2}$} & \multicolumn{2}{|c|}{$\mathrm{HF}$} & \multicolumn{2}{|c|}{$\mathrm{CO}$} & \multicolumn{2}{|c|}{$F_{2}$} \\
\hline & DZP & TZ2P & $\mathrm{DZP}$ & TZ2P & $\mathrm{DZP}$ & TZ2P & $\mathrm{DZP}$ & $\mathrm{TZ2P}$ & $\mathrm{DZP}$ & $\overline{T Z 2 P}$ \\
\hline B-LYP & 0.748 & 0.743 & 1.128 & 1.104 & 0.937 & 0.933 & 1.156 & 1.137 & 1.431 & 1.440 \\
\hline B-LYP/HF & 0.748 & 0.742 & 1.123 & 1.099 & 0.932 & 0.929 & 1.149 & 1.130 & 1.417 & 1.426 \\
\hline $\mathrm{K}_{\tau} \mathrm{B}-\mathrm{L} Y \mathrm{P}$ & 0.752 & * & 1.067 & * & 0.875 & $*$ & 1.099 & $*$ & 1.294 & $*$ \\
\hline $\mathrm{K}_{\mathrm{T}} \mathrm{B}-\mathrm{L} \mathrm{YP} / \mathrm{HF}$ & 0.747 & 0.742 & 1.067 & 1.051 & 0.873 & 0.877 & 1.099 & 1.086 & 1.293 & 1.298 \\
\hline experiment ${ }^{30}$ & \multicolumn{2}{|c|}{0.742} & \multicolumn{2}{|c|}{1.098} & \multicolumn{2}{|c|}{0.917} & \multicolumn{2}{|c|}{1.128} & \multicolumn{2}{|c|}{1.412} \\
\hline
\end{tabular}

1988 correction,

$$
\begin{aligned}
E_{\mathrm{x}}^{\mathrm{B}}=-\frac{-3}{4}\left(\frac{3}{\pi}\right)^{1 / 3} \int \varrho^{4 / 3}(\mathbf{r}) \mathrm{d} \mathbf{r}- & \\
& \sum_{\sigma} \int \beta \varrho_{\sigma}^{4 / 3} \frac{x_{\sigma}{ }^{2}}{\left(1+6 \beta x_{\sigma} \sinh ^{-1} x_{\sigma}\right)} \mathrm{d} \mathbf{r}
\end{aligned}
$$

to the exact exchange energies of the six noble gas atoms $\mathrm{He}$ to $\mathrm{Rn}$. We therefore suggest two new exchange functionals: $E_{\mathrm{x}}^{\mathrm{G}}$ and $E_{\mathrm{x}}^{\mathrm{T}}$.

$$
\begin{aligned}
E_{\mathrm{x}}^{\mathrm{G}}=\frac{-3}{4 \pi} \int \frac{\varrho^{3}(\mathbf{r})}{t} \mathrm{~d} \mathbf{r}- & \\
& \sum_{\sigma} \int \beta_{\mathrm{G}_{\sigma}{ }^{4 / 3}} \frac{x_{\sigma}^{2}}{\left(1+6 \beta_{\mathrm{G}} x_{\sigma} \sinh ^{-1} x_{\sigma}\right)} \mathrm{d} \mathbf{r}
\end{aligned}
$$

$$
\begin{aligned}
E_{\mathrm{x}}^{\mathrm{T}}=\frac{-27}{40 \pi} \int \frac{Q^{3}(\mathbf{r})}{t} & \mathrm{~d} \mathbf{r}- \\
& \sum_{\sigma} \int \beta_{\mathrm{T}} \varrho_{\sigma}^{4 / 3} \frac{x_{\sigma}^{2}}{\left(1+6 \beta_{\mathrm{T}} x_{\sigma} \sinh ^{-1} x_{\sigma}\right)} \mathrm{d} \mathbf{r}
\end{aligned}
$$

where $\beta_{\mathrm{G}}$ and $\beta_{\mathrm{T}}$ are two parameters to be obtained by fitting eqs 71 and 72 to the exchange energies of the noble gas atoms. We determined the values of $\beta_{\mathrm{G}}$ and $\beta_{\mathrm{T}}$ to be 0.0015 and 0.009 , respectively; the results of the fits are presented in Table 3. The functional $E_{\mathrm{x}}^{\mathrm{G}}$ appears to be more dependent on the atomic number than $E_{\mathrm{x}}^{\mathrm{T}}$, with $E_{\mathrm{x}}^{\mathrm{T}}$ giving a significantly better fit. We note that the original $E_{\mathrm{x}}^{\mathrm{B}}$ exchange functional gives by far the best fit to the Hartree-Fock exchange energies. In Figures 7 and 8 we compare the potential energy curves calculated using $E_{\mathrm{x}}^{\mathrm{HF}}, E_{\mathrm{x}}^{\mathrm{B}}, E_{\mathrm{x}}^{\mathrm{G}}$, and $E_{\mathrm{x}}^{\mathrm{T}}$ for the $\mathrm{H}_{2}$ and $\mathrm{N}_{2}$ molecules, respectively. We observe from Figure 7 that neither $E_{\mathrm{x}}^{\mathrm{G}}$ or $E_{\mathrm{x}}^{\mathrm{T}}$ is as good a predictor as $E_{\mathrm{x}}^{\mathrm{B}}$ of the Hartree-Fock exchange for the $\mathrm{H}_{2}$ molecule in the vicinity of the equilibrium geometry. The corresponding potential energy curves for the $\mathrm{N}_{2}$ molecule are given in Figure 8. Again we find that, in the region of the equilibrium geometry, $E_{\mathrm{x}}^{\mathrm{B}}$ gives a better reproduction of the Hartree-Fock exchange than either $E_{\mathrm{x}}^{\mathrm{G}}$ or $E_{\mathrm{x}}^{\mathrm{T}}$.

In the final stage of our investigation, we augmented the exchange energy functionals $E_{\mathrm{x}}^{\mathrm{B}}$ and $E_{\mathrm{x}}^{\mathrm{T}}$ with an appropriate correlation energy functional and optimized the bond lengths for the following molecules: $\mathrm{H}_{2}, \mathrm{~N}_{2}, \mathrm{HF}, \mathrm{CO}$, and $\mathrm{F}_{2}$. We chose the LYP correlation functional due to Lee, Yang, and Parr $E_{\mathrm{c}}^{\text {LYP } 6,7}$ because it has been established that the use of the functional $E_{\mathrm{x}}^{\mathrm{B}}$ in combination with $E_{\mathrm{c}}^{\mathrm{LYP}}$ predicts both repectable bond lengths ${ }^{11,12}$ and thermochemical properties ${ }^{29}$ for small molecules (this particular combination of the functionals, $E_{\mathrm{xc}}$ $=E_{\mathrm{x}}^{\mathrm{B}}+E_{c}^{L Y P}$, is commonly denoted B-LYP). On augmenting the new functional $E_{\mathrm{x}}^{\mathrm{T}}$ with $E_{\mathrm{c}}^{\mathrm{LYP}}$, we arrive at a new form for the exchange-correlation functional $E_{\mathrm{xc}}$, and we denote this as $\mathrm{K}_{\mathrm{T}} \mathrm{B}-\mathrm{LYP}$. We optimized the bond lengths using the B-LYP and $\mathrm{K}_{\mathrm{T}} \mathrm{B}-\mathrm{LYP}$ forms for the exchange-correlation functional. The results for the set of molecules mentioned above, with both DZP and TZ2P basis sets, are presented in Table 4. With the exception of $\mathrm{H}_{2}$ and $\mathrm{HF}$, we find that all the bond lengths calculated with $\mathrm{K}_{\mathrm{T}} \mathrm{B}$-LYP are consistently too short; this is in contrast to B-LYP, where the bond lengths are too large. Increasing the basis set size from DZP to TZ2P does not appear to improve matters.

\section{Conclusion}

In this paper we have investigated two alternative exchange functionals, originally suggested by Lee and Parr, namely, $K_{\mathrm{G}}$ and $K_{\mathrm{T}}$. They are derived through a quadratic expansion of $\mathbf{r}_{1}$ and $\mathbf{r}_{2}$ in $\left|\mathbf{r}_{1}-\mathbf{r}_{2}\right|$ about the center of mass $(1 / 2)\left(\mathbf{r}_{1}+\mathbf{r}_{2}\right)$. In particular, $K_{\mathrm{G}}$ is a functional which is not related to the uniform electron gas approximation, although $K_{\mathrm{T}}$, being derived through Bessel functions, does have some relation. We selected $\mathrm{H}_{2}$ and $\mathrm{N}_{2}$ for detailed investigations and performed calculations on $\mathrm{F}_{2}$, $\mathrm{CO}$, and $\mathrm{HF}$ as well.

Our results show that $K_{\mathrm{G}}$ and $K_{\mathrm{T}}$, and other variants of them which we also investigated, do not offer promising alternative starting points to the Dirac uniform electron gas exchange functional. In particular, by comparing Hartree-Fock bond lengths, predictions using $K_{\mathrm{G}}$ and $K_{\mathrm{T}}$ are, with the exception of $\mathrm{H}_{2}$ and $\mathrm{HF}$, much shorter. We were unable to "fix up" $K_{\mathrm{G}}$ and $K_{\mathrm{T}}$ satisfactorily using a Becke type gradient correction functional, and so the exchange energies of the noble gas atoms were not well reproduced. Indeed, it is surprising how well the Dirac LDA exchange term can be "fixed up" by Becke's 1988 gradient correction. Thus, energies obtained using the functionals $K_{\mathrm{G}}$ and $K_{\mathrm{T}}$ are in some error. The use of the LDA as a starting point for the derivation of good density functionals may be difficult to improve upon.

Note Added in Proof: A late referee's report made some important observations:

(1) When relative and center-of-mass coordinates are used, there is no local hole normalization constraint, as there is if one uses the relative coordinate and $r_{1}$. A local normalization constraint is obviously quite restrictive and is therefore of great value. Becke and Roussel (Phys. Rev. 1989, A39, 3761) have introduced an exchange functional which does obey the local hole normalization constraint. Global rescaling is not an acceptable fix because it will give spurious dissociation limits in heteronuclear molecules.

(2) For the Gaussian and Bessel models to make sense, $t(\mathbf{r}, \varrho)$ must be positive, because the models are not flexible enough to accommodate curvatures of either sign. The authors have found points at which $t(\mathbf{r}, \varrho)$ is negative. This highlights a serious flaw in the model.

The referee considers that these shortcomings, the effects of which are demonstrated in the paper, are serious criticisms of the models. He therefore supported the conclusions of the paper. 


\section{References and Notes}

(1) Hohenberg, P.; Kohn, W. Phys. Rev. 1964, B136, 864

(2) Kohn, W.; Sham, L. J. Phys. Rev. 1965, A140, 1133.

(3) Dirac, P. A. M. Proc. Cambridge Phil. Soc. 1930, 26, 376

(4) Vosko, S. J.; Wilk, L.; Nusair, M. Can. J. Phys. 1980, 58, 1200.

(5) Becke, A. D. Phys. Rev. 1988, A38, 3098. Becke, A. D. In The Challenge of $d$ and felectrons, Theory and Computation; Salahub, D. R. Zerner, M. C., Eds.; ACS Symposium Series 394; 1989.

(6) Lee, C.; Yang, W.; Parr, R. G. Phys. Rev. 1988, B37, 785.

(7) Miehlich, B.; Savin, A.; Stoll, H.; Preuss, H. Chem. Phys. Lett. 1989, 157,200

(8) Perdew, J. P. Phys. Rev. 1986, B33, 8822.

(9) Perdew, J. P. Electronic Structure of Solids '91; Ziesche, P., Eschring, H., Eds.; Akademie Verlag: Berlin, 1991.

(10) Johnson, B. G.; Gill, P. M. W.; Pople, J. A. J. Chem. Phys. 1992, $97,7846$.

(11) Murray, C. W.; Laming, G. J.; Handy, N. C.; Amos, R. D. Chem. Phys. Lett. 1992, 199, 551.

(12) Laming, G. J.; Handy, N. C.; Amos, R. D. Molec. Phys. 1993, 80 , 1121.

(13) Becke, A. D. J. Chem. Phys. 1993, 98, 1372

(14) Becke, A. D. J. Chem. Phys. 1993, 98,5648

(15) Parr, R. G.; Yang, W. Density Functional Theory of Atoms and Molecules; Oxford: New York, 1989.

(16) Johnson, B. G.; Gill, P. M. W.; Pople, J. A. J. Chem. Phys. 1993, 98,5612 .
(17) Laming, G. J.; Termath, V.; Handy, N. C. J. Chem. Phys. 1993, 99,8765

(18) Andzelm, J.; Wimmer, E. J. Chem. Phys. 1992, 96, 1280.

(19) Ghosh, S. K.; Parr, R. G. Phys. Rev. 1986, A34, 2377.

(20) Lee, C.; Parr, R. G. Phys. Rev. 1987, A35, 785.

(21) Ghosh, S. K.; Berkowitz, M.; Parr, R. G. Proc. Natl. Acad. Sci. U.S.A. 1984, 81, 8028.

(22) Kemister, G, Phys, Rev. 1986, A34, 4480

(23) Berkowitz, M. Chem. Phys. Lett. 1986, 129, 486.

(24) Meyer, J.; Bartel, J.; Brack, M.; Quentin, P.; Aicher, S. Phys. Lett. 1986, B172, 122

(25) Amos, R. D.; Alberts, I. L.; Andrews, J. S.; Colwell, S. M.; Handy, N. C.; Jayatilaka, D.; Knowles, P. J.; Kobayashi, R.; Koga, N.; Laidig, K. E.; Maslen, P. E.; Murray, C. W.; Rice, J. E.; Sanz, J.; Simandiras, E. D.; Stone, A. J.; Su, M.-D. CADPAC5: The Cambridge Analytic Derivatives Package; Cambridge, 1992.

(26) Murray, C. W.; Handy, N. C.; Laming, G. J. Mol. Phys. 1993, 78, 997.

(27) Dunning, T. H. J. Chem. Phys. 1970, 53, 2823.

(28) Dunning, T. H. J. Chem. Phys. 1971, 55, 716.

(29) Gill, P. M. W.; Johnson, B. G.; Pople, J. A.; Frisch, M. J. J. Chem. Phys. 1992, 197, 499.

(30) Huber, K. P.; Herzberg, G. Constants of Diatomic Molecules; Van Nostrand-Reinhold: New York, 1979.

(31) Kolos, W.; Roothaan, C. C. J. Phys. Rev. 1960, 32, 219.

JP9418300 